\title{
The method of Yiqi Yangyin Tongluo can attenuate the pyroptosis of rheumatoid arthritis chondrocytes through the ASIC1a/NLRP3 signaling pathway
}

\author{
Yeyu Zhao ${ }^{1,2 \#}$, Yiran Chen ${ }^{2 \#}$, Zhimin Wang ${ }^{3}$, Cheng Peng ${ }^{4}$, Quansheng $\mathrm{Li}^{5}$, Jin Wu ${ }^{6}$, Linmin Wang ${ }^{1}$, \\ Dongwu Liu ${ }^{1}$, Yue Yue ${ }^{1}$, Qi Qing ${ }^{1}$, Pengyuan Sun ${ }^{1}$, Liang Liang ${ }^{7}$, Huan Yu ${ }^{8}$, Xing Ju ${ }^{5}$, Lue Li $^{1}$, \\ Enlong Wang ${ }^{1}$, Mingli Gao ${ }^{1}$, Jing Yu ${ }^{1}$, Tieming $\mathrm{Ma}^{2}$
}

${ }^{1}$ Department of Rheumatology, Affiliated Hospital of Liaoning University of Traditional Chinese Medicine, Shenyang, China; ${ }^{2}$ School of Acupuncture, Moxibustion and Tuina, Liaoning University of Traditional Chinese Medicine, Shenyang, China; ${ }^{3}$ Department of Endocrinology, Affiliated Hospital of Liaoning University of Traditional Chinese Medicine, Shenyang, China; ${ }^{4}$ Department of Laboratory, Liaoning University of Traditional Chinese Medicine Affiliated Hospital, Shenyang, China; ${ }^{5}$ Department of Allergy, Shengjing Hospital of China Medical University, Shenyang, China; ${ }^{6}$ Liaoning University of Traditional Chinese Medicine Innovation Engineering Technology Center of Traditional Chinese Medicine, Shenyang, China; ${ }^{7}$ Department of Nephrology, Affiliated Hospital of Liaoning University of Traditional Chinese Medicine, Shenyang, China; ${ }^{8}$ Department of Ultrasound, Affiliated Hospital of Liaoning University of Traditional Chinese Medicine, Shenyang, China

Contributions: (I) Conception and design: Y Zhao, Y Chen, T Ma; (II) Administrative support: J Yu; (III) Provision of study materials or patients: Y Zhao; (IV) Collection and assembly of data: All authors; (V) Data analysis and interpretation: Y Zhao; (VI) Manuscript writing: All authors; (VII) Final approval of manuscript: All authors.

\#These authors contributed equally to this work.

Correspondence to: Jing Yu. Department of Rheumatology, Affiliated Hospital of Liaoning University of Traditional Chinese Medicine, Shengyang 110032, China. Email: yujinglnzyfsk@163.com; Tieming Ma. School of Acupuncture, Moxibustion and Tuina, Liaoning University of Traditional Chinese Medicine, Shenyang, China. Email: matieming999@sohu.com.

Background: Based on the ASIC1a/NLRP3 signaling pathway, we explored the specific molecular mechanism of the pyroptosis of rheumatoid arthritis (RA) chondrocytes by the method of nourishing qi, nourishing yin, and dredging collaterals to provide new ideas for the treatment of this disease.

Methods: A total of 50 rats were divided into a normal group, model group, methotrexate group, Yiqi Yangyin Tongluo group, and combined group. Except for the normal control group, the other groups used Freund's complete adjuvant (FCA) to make RA rat model. The arthritis index and ankle joint swelling of rats in each group were recorded. HE staining and ELISA were used to assess the pathology of the ankle joint of each group of rats and the content of IL- $1 \beta$ and IL-18 in rat serum. Furthermore, immunofluorescence and qPCR methods were used to detect the protein and mRNA expression levels of NLRP3, caspase 1, ACS, and ASIC1a in the cartilage tissue of each group of rats.

Results: Compared with the normal group, the right hind foot joint of the model group was significantly swollen, the levels of IL-18 and IL-1 $\beta$ in the serum of rats increased significantly, and the mRNA and protein levels of NLRP3, caspase 1, ACS, and ASIC1a in the chondrocytes also increased significantly. Compared with the model group, the degree of ankle joint swelling and IL-18 and IL-1 $\beta$ content in rat serum in each medication group was significantly reduced, and the combined group showed the greatest reduction compared with the other groups. After 8 weeks of treatment, compared with the model group, the mRNA and protein levels of NLRP3, caspase 1, ACS, and ASIC1a in the chondrocytes of each medication group were down-regulated. HE staining found that there were large numbers of infiltrating inflammatory cells and pannus in the joint tissue of the model group, while only a small amount of inflammatory cell infiltration and pannus was seen in the joint tissue of the rats in each treatment group.

Conclusions: The method of Yiqi Yangyin Tongluo can attenuate the pyroptosis of RA chondrocytes through the ASIC1a/NLRP3 signaling pathway. 
Keywords: The method of Yiqi Yangyin; rheumatoid arthritis (RA); ASIC1a/NLRP3 signaling pathway; cell pyroptosis; methotrexate

Submitted Nov 25, 2021. Accepted for publication Jan 19, 2022.

doi: $10.21037 / \mathrm{atm}-21-6822$

View this article at: https://dx.doi.org/10.21037/atm-21-6822

\section{Introduction}

Rheumatoid arthritis (RA) is a chronic autoimmune disease with multiple joint involvements as the predominant manifestation. Its main characteristics are chronic synovial inflammation, cartilage degeneration, and joint destruction. The prevalence in China is about $0.32-0.36 \%$ (1). The disability rate of RA patients 1 year after its onset is as high as $20 \%(2-4)$. The disease activity and clinical remission rate of RA patients in China are far lower than expected. At present, the therapeutic drugs mainly include hormone drugs, non-steroidal anti-inflammatory drugs, and biological agents. The efficacy of traditional Chinese medicine for the treatment of RA has been affirmed. It can cooperate with western medicine to relieve the condition, improve the clinical remission rate, reduce the adverse reactions of western medicine interventions, and improve the quality of life of patients. Current studies suggest that if the course of RA is more than 5 years or after glucocorticoid treatment (5), it is prone to manifestations of deficiency of both qi and yin $(6,7)$. These findings cannot be explained by the previous classification of syndrome types. Based on the theory of the method of Yiqi Yangyin and many years of clinical evidence, the final established treatment is the prescription of Yiqi Yangyin Tongluo, and relieving pain-Sishenjian. Sishenjian was described in Bao Xiang's New Compilation of Prescriptions in the Qing Dynasty. New Compilation of Prescriptions states: "When the disease lies in the tendons, it cannot be flexed, while in the bones, it is difficult to move. After a long period, it will be thick and swollen every day, and the thighs will become thin every day. Pain without pus, unchanged color, success, or failure. Sishenjian was established." (8). The prescription has the effects of replenishing qi, nourishing yin, dredging collaterals, and relieving pain, and can improve the symptoms of RA patients such as joint pain, morning stiffness, fatigue, and secondary dry mouth and eyes. The curative effect has shown to be significant across a large number of clinical applications. It can not only improve the symptoms of patients and increase the clinical remission rate, but it can also improve the quality of life of patients.

In recent years, a type of programmed cell necrosis in RA bone destruction has been found, namely pyroptosis (9-12). Pyrolysis belongs to programmed necrosis and is related to inflammation. The deficiency of qi and yin belongs to the syndrome of the original deficiency and the superficiality, and the deficiency of qi and yin can produce pathological products and cause inflammation over time. Deficiency of both qi and yin is actually the proof of the deficiency of the original body and the correctness of the essence. Long-term deficiency of Qi and Yin can cause pathological products such as deficiency heat, water dampness, phlegm and blood stasis. These pathological products can lead to chronic inflammation, which is consistent with programmed necrosis of pyrolysis. The procedural necrosis here is chronic and continuous, which is also an important reason why RA is not easy to control. The classical activation pathway of pyroptosis is mainly mediated by the NLRP3 inflammasome. By activating cysteine aspartate proteolytic enzyme 1 (caspase 1), it leads to the release of inflammatory factors, thereby inducing a cascade of inflammatory responses. It has an important regulatory effect on the body's immune response (13). The NLRP3 inflammasome, composed of the NLR family, is one of the most widely studied inflammasomes. It is involved in the pathogenesis of inflammation and tumors, and also plays an important role in the maintenance of normal physiological homeostasis. The NLRP3 inflammasome can also be activated by various types of stimuli, making it one of the most widely studied inflammasomes $(14,15)$. After the NLRP3 inflammasome is stimulated by the external environment, the C-terminus of the receptor protein NLRP3 binds to the ligand, and the $\mathrm{N}$-terminus recruits the downstream linker molecule ASC and the precursor of the effector molecule caspase 1 . After the 3 are assembled, they can promote the shearing and activation of the precursor of caspase 1. At the same time, studies have shown that (16) caspase 1 can cut the common substrate gasdermin D (GSDMD) of inflammatory caspases to release the active $\mathrm{N}$-terminal (GSDMD-N), induce cell membrane perforation, promote the maturation and 
secretion of IL- $1 \beta$ and IL-18, and also cause the cell pyroptosis. In the joints of RA patients, IL- $1 \beta$ rapidly attenuates proteoglycan by inducing the production of matrix metalloproteinases and proteoglycanase to degrade cartilage and inhibit the repair of bone and cartilage. It plays a key role in the destruction of bone and cartilage in RA patients. IL-18 can induce macrophages to produce GM-CSF, NO, TNF- $\alpha$, and other substances, which participate in the inflammatory activity of RA, and TNF- $\alpha$ and IL- $1 \beta$ can also significantly increase the expression of IL-18 $(17,18)$.

Acid-sensing ion channels (ASICs) belong to the ENaC/DEG channel family (19). The characteristics of ASICs and other members of the family are encoded by 4 functional genes, including ASIC1, ASIC2, ASIC3, and ASIC4. So far, 7 subunits have been cloned (ASIC1a, ASIC1b, ASIC1b2, ASIC2a, ASIC2b, ASIC3, ASIC4) (20). The subunits of ASICs are distributed in different tissues. ASIC1a and ASIC2a are widely distributed in the central and peripheral nervous systems. In recent years, research on ASICs has progressed rapidly. ASIC1a has a wide range of biological functions and participates in the pathological processes of many diseases, and has therefore become a research focus. It is mainly distributed in the central cerebral cortex, cerebellum, hypothalamus, hippocampus, spinal cord, and peripheral neurons, and is also expressed in cartilage and bone tissues. ASIC1a plays an important role in many pathological processes associated with tissue acidification diseases, such as cerebral hypoxia-ischemia, epilepsy, inflammation, and tumors, among others, and one of the important pathological features of RA is local tissue acidification. Reactive oxygen species (ROS) are important second messenger molecules in cells. ROS have extremely important biological activities and functions, and play an important role in the occurrence and development of various diseases (21). The production of ROS, the outflow of potassium ions, changes in cell volume, calcium signaling, and lysosomal interference are all considered to be the key upstream signals which activate NLRP3 (22-25).

At present, there is still a lack of research on the underlying mechanism of Yiqi Yangyin Tongluo method as an intervention for RA chondrocyte pyroptosis. Some studies have found that ASIC1a is expressed in articular chondrocytes of RA rats. Furthermore, ASIC1a was shown to regulate extracellular $\mathrm{Ca}^{2+}$ influx, causing joint $\mathrm{Ca}^{2+}$ overload in chondrocytes and the up-regulation of ROS expression in articular chondrocytes, which increases the expression of NLRP3, and finally induces pyroptosis of articular chondrocytes $(26,27)$. To this end, this study conducted in vivo animal experiments to clarify the therapeutic mechanism of the Yiqi Yangyin Tongluo method through the ASIC1a/NLRP3 signaling pathway to improve chondrocyte pyroptosis. This work provides a basis for the treatment of RA with the method of nourishing qi, nourishing yin, and dredging collaterals, and also provides more ideas for improving the clinical remission rate of RA using traditional Chinese medicine.

We present the following article in accordance with the ARRIVE reporting checklist (available at https://atm. amegroups.com/article/view/10.21037/atm-21-6822/rc).

\section{Methods}

\section{Experimental animals}

A total of 50 male specific-pathogen free (SPF) Sprague Dawley (SD) rats (6 months old, female, weighing 180-220 g) were purchased from Beijing Weitong Lihua Biotechnology Co., Ltd. The rats were raised in the SPF laboratory of the Experimental Animal Center of Liaoning University of Traditional Chinese Medicine. The laboratory temperature was controlled at $22 \pm 2{ }^{\circ} \mathrm{C}$, the laboratory humidity was controlled at $45 \% \pm 5 \%$, and the natural light cycle was used. Experiments were performed under a project license (No. SCXK 2019-0008) granted by the Experimental Animals Ethics Committee of the Affiliated Hospital of Liaoning University of Traditional Chinese Medicine, in compliance with national and the Affiliated Hospital of Liaoning University of Traditional Chinese Medicine guidelines for the care and use of animals.

\section{Main reagents}

The Chinese medicine decoction components were all purchased at one time from the Pharmacy Bureau of the Affiliated Hospital of Liaoning University of Traditional Chinese Medicine and were identified as genuine products by the Department of Traditional Chinese Medicine of Liaoning University of Traditional Chinese Medicine. The composition and dosage of the compound of Yiqi Yangyin Tongluo medicine used in the experiment were as follows: $30 \mathrm{~g}$ of raw astragalus, $15 \mathrm{~g}$ of dendrobium, $15 \mathrm{~g}$ of polygala, $25 \mathrm{~g}$ of achyranthes, $15 \mathrm{~g}$ of honeysuckle. Aspirin entericcoated tablets $(25 \mathrm{mg} \times 100$ tablets) were purchased from Nanjing Baijingyu Pharmaceutical Co. Ltd. Methotrexate were made by grinding aspirin enteric-coated tablets into a 
fine powder, adding to distilled water, and stirring.

Freund's complete adjuvant (FCA; 7024) was purchased from Chondrex, USA. Dimethyl sulfoxide (DMSO) was purchased from Sigma (USA). The fluorescent secondary antibody was purchased from abcam (USA). Rat IL-1 $\beta$ and IL-18 enzyme-linked immuno-absorbent assay (ELISA) kits were purchased from Wuhan Elab science company. Trypsin, penicillin-streptomycin double-antibody, RIPA protein lysate, BCA protein concentration determination kit, Lipofectamine 2000, and Trizol were purchased from Invitrogen. The reverse transcription kit was provided by Dalian Bao Biological Company. RNA primers were synthesized by Dalian Bao Biological Company. TBS buffer was provided by Boster company. ASIC1a antibody was purchased from Alomone Labs. Cleaved caspase-1p10, ASC, and NLRP3 antibodies were purchased from Abcam, USA.

\section{Main instruments}

The precision $\mathrm{PH}$ meter PHS3C was purchased from Shanghai Leici Instrument Factory. The FA2004 electronic analytical balance is the product of Shanghai Jingtian Balance Instrument Factory. Microtome is the product of German Leica Company. The toe volume measuring instrument YLSTA is the product of the equipment station of Shandong Academy of Medical Sciences. The Millipore water purifier was purchased from Pall, USA. The clean workbench SW CJ 1F was from Sujing Group Suzhou Antai Air Technology Co., Ltd. The real-time PCR amplification instrument was purchased from Shanghai Thermo Company. The reverse transcription PCR amplification instrument was purchased from Eppendorf, Germany. The 5415R high-speed refrigerated centrifuge was purchased from Eppendorf, Germany. The BX50 optical microscope was purchased from Olympus.

\section{Experimental method}

\section{Model preparation and animal grouping}

SD rats were injected intracutaneously with $100 \mu \mathrm{L}$ of FCA (20 $\mathrm{mg} / \mathrm{mL}$ ) in the right hind toe, and the control group was injected with the same amount of normal saline. Swelling of the feet on the primary side and the secondary side of the rat were observed. From the first day of adjuvant injection, the swelling of the limbs of SD rats was observed daily. The sum of the scores of the limbs was the arthritis index (AI), and the higher the AI, the more severe the arthritis damage. One week after injection, the AI score was calculated, and if $\mathrm{AI} \geq 4$, the modeling was considered successful, while rats with insufficient AI scores were not used.

A total of $50 \mathrm{SD}$ rats were randomly divided into a normal group, model group, compound of Yiqi Yangyin Tongluo group (Yiqi Yangyin Tongluo group), positive control methotrexate group, and combined group (Yiqi Yangyin Tongluo combined with methotrexate). In the third week (d14-21) after modeling, rats in the methotrexate group were given methotrexate $1.89 \mathrm{mg} / \mathrm{kg} /$ week by gavage. Rats in the Yiqi Yangyin Tongluo group were given compound of Yiqi Yangyin Tongluo soup and the dosage for rats was $3 \mathrm{~mL} /$ day. The liquid was decocted and concentrated to a crude drug concentration of $0.6 \mathrm{~g} / \mathrm{mL}$ once a day by gavage. The rats in the model group were given the same amount of normal saline. The swelling degree of the secondary paws of the rats in each group were measured on the 0th, 10th, 14th, 21 st, and 28th days after the model was established, and pictures were taken. All rats were sacrificed at the 8th week after administration, and the plasma, spleen, and ankle joints of rats in each group were collected.

\section{Dosing schedule}

According to the ratio between human and rat body surface area, the equivalent dose was calculated (1), and the conversion factor for adults and rats is 6.3. The total weight of compound of Yiqi Yangyin Tongluo medicinal materials was $100 \mathrm{~g}$. Methotrexate was calculated according to the instructions. Rats in methotrexate group were given $1.89 \mathrm{mg} / \mathrm{kg} /$ week by gavage, and the average weight of the rats was $200 \mathrm{~g}$. The dosage calculations were as follows:

* Normal group: $10 \mathrm{~mL} / \mathrm{kg} / \mathrm{d}$ of sterile water for injection daily, gavage once a day; Model group: $10 \mathrm{~mL} / \mathrm{kg} / \mathrm{d}$ of sterile water for injection daily, gavage once a day;

* Yiqi Yangyin Tongluo group: Due to the large dosage of the original prescription, through the clinical practice and research of this research group, the drug dosage of the original prescription of Sishen Jian was re-approved, and finally the "Yiqi Yangyin Tongluo" was formed. The dosage of the prescription is: raw astragalus $30 \mathrm{~g}$, dendrobium $15 \mathrm{~g}$, polygala $15 \mathrm{~g}$, achyranthes bidentata $25 \mathrm{~g}$, honeysuckle $15 \mathrm{~g}$. The total weight of compound of Yiqi Yangyin Tongluo medicinal materials was $100 \mathrm{~g}$. The dosage for adults was $100 \mathrm{~g} / 70 \mathrm{~kg} / \mathrm{d}$, and the conversion coefficient between adults and rats is 6.3. The average body weight of rats is $200 \mathrm{~g}$, so the dosage of the compound of Yiqi Yangyin Tongluo low-dose group was 
$4.5 \mathrm{~g} / \mathrm{kg} / \mathrm{d}$, the dosage of the compound of Yiqi Yangyin Tongluo middle-dose group was $9 \mathrm{~g} / \mathrm{kg} / \mathrm{d}$, and the dosage of the compound of Yiqi Yangyin high-dose group was $18 \mathrm{~g} / \mathrm{kg} / \mathrm{d}$;

- Methotrexate group: According to the conversion coefficient between adults and rats (6.3), rats in the methotrexate group were given $1.89 \mathrm{mg} / \mathrm{kg} /$ week of methotrexate by intragastric administration;

* Combined group (Yiqi Yangyin Tongluo + methotrexate group): same as above.

\section{Main indexes and detection methods}

Observation of general biological signs and the body weight, activity level, reaction, spirit, fur, stool, death, and other symptoms and signs of rats were observed every day.

\section{The AI score evaluates joint inflammation}

The rats were scored after the injection of drugs to induce inflammation. The evaluation standard is a 5 -level scoring method to record the occurrence and severity of arthritis lesions in the extremities of rats: Grade 0: no arthritis, 0 points; Grade 1: individual feet with redness and swelling of the small toe joints, 1 point; Grade 2: most toes and feet are red and swollen, 2 points; Grade 3: ankle redness and swelling below the joints, 3 points; Grade 4: redness and swelling below the ankle joints and above the ankle joints, 4 points. The scores of each joint of the limbs of the rats were recorded and totaled, which was the AI, with a minimum score of 0 and a maximum score of 16 .

\section{Measurement and calculation of the swelling degree of the right foot}

In the experiment, a toe volume measuring instrument was used to determine the right posterior ankle joint of the rat as the baseline, and a marker was used to mark the position. The volume of the right hind foot of each rat was measured before modeling and recorded as V0. After the injectioninduced inflammation and drug intervention started, the volume of the rat's foot was measured at day 24, 52, and 80 , and recorded as $\mathrm{Vt}$. The degree of joint swelling $(\%)=$ $(\mathrm{Vt}-\mathrm{V} 0) / \mathrm{V} 0 \times 100 \%$ was calculated, each rat was measured 3 times each time, and the average value was calculated.

\section{Hematoxylin and eosin (HE) staining of the rat ankle joint} For pathological examination of the rat ankle joint, the excess fur and muscle tissue around the rat ankle joint was removed, and the joint was rinsed with normal saline to remove the debris on the joint surface. After fixing all joints with $4 \%$ neutral paraformaldehyde solution for $48 \mathrm{~h}$, they were decalcified with $10 \%$ neutral EDTA solution for 2 months, during which they were replaced with fresh EDTA solution twice a week. After dehydration with different concentrations of ethanol solution, paraffin embedding, sectioning $(7 \mu \mathrm{m})$, and xylene dewaxing, the tissues were soaked with hematoxylin solution/ eosin solution and finally sealed with neutral gum. The pathological changes of the ankle joints of rats in each group were observed under a microscope.

\section{Immunofluorescence (IF)}

Rat ankle joint sections were stained with the above HE staining procedure. After the tissue sections were deparaffinized with xylene, the effect of endogenous peroxidase was eliminated with $3 \% \mathrm{H}_{2} \mathrm{O}_{2}$. The tissue antigens were retrieved using antigen retrieval solution, and then blocked with goat serum. Subsequently, ASIC1a, NLRP3, caspase 1, and ASC specific antibodies were added and incubated at $4{ }^{\circ} \mathrm{C}$ for $12 \mathrm{~h}$ (PBS buffer replaced the primary antibody as a negative control). Then, a fluorescent secondary antibody (Invitrogen, USA) was used at room temperature. The cells were washed three times with PBS, 15 min each. The expression of ASIC1a, NLRP3, caspase 1, and ASC in the articular cartilage of the ankle joint of each group was observed under a microscope.

\section{Cytokine detection}

Serum IL- $1 \beta$ and IL-18 were detected by the enzyme-linked immunosorbent assay (ELISA method. Rat IL- $1 \beta$ and IL18 ELISA kits were purchased from Wuhan Elab science company, and the kit instructions were followed for testing.

\section{Real-time quantitative PCR}

Rat knee cartilage tissues were collected from different administration groups, model groups, and normal groups, and Trizol (Invitrogen) was used to extract total RNA from the tissues. A total of $2 \mu \mathrm{g}$ RNA was used for reverse transcription to synthesize the first strand of cDNA, and the reaction system of qPCR $(20 \mu \mathrm{L})$ contained the following: SYBR-Green mix $10 \mu \mathrm{L}$, upstream and downstream primers $(10 \mu \mathrm{mol} / \mathrm{L})$ each $1 \mu \mathrm{L}, 10$ times diluted cDNA template $2 \mu \mathrm{L}$, ultrapure water $6 \mu \mathrm{L}$. The qPCR experiment was performed on the LightCycle93 (Roche, Switzerland) fluorescence quantitative PCR instrument. The human $\beta$-actin gene was used as an internal reference, and 3 technical replicates and 3 biological replicates were used. 
Table 1 RT-PCR primer list

\begin{tabular}{lll}
\hline Gene & Forward primer $\left(5^{\prime}-3^{\prime}\right)$ & Reverse primer $\left(5^{\prime}-3^{\prime}\right)$ \\
\hline ASIC1a & GGCCAACTTCCGTAGCTTCA & ATGCCCTGCTCTGTCGTAGAA \\
NLRP3 & CAGACCTCCAAGACCACGACTG & CATCCGCAGCCAATGAACAGAG \\
Caspase 1 & TGCCTGGTCTTGTGACTTGGAG & ATGTCCTGGGAAGAGGTAGAAACG \\
ASC & TTATGGAAGAGTCTGGAGCTGTGG & AATGAGTGCTTGCCTGTGTTGG \\
$\beta$-actin & GGAGATTACTGCCCTGGCTCCTA & GACTCATCGTACTCCTG CTTGCTG \\
\hline
\end{tabular}
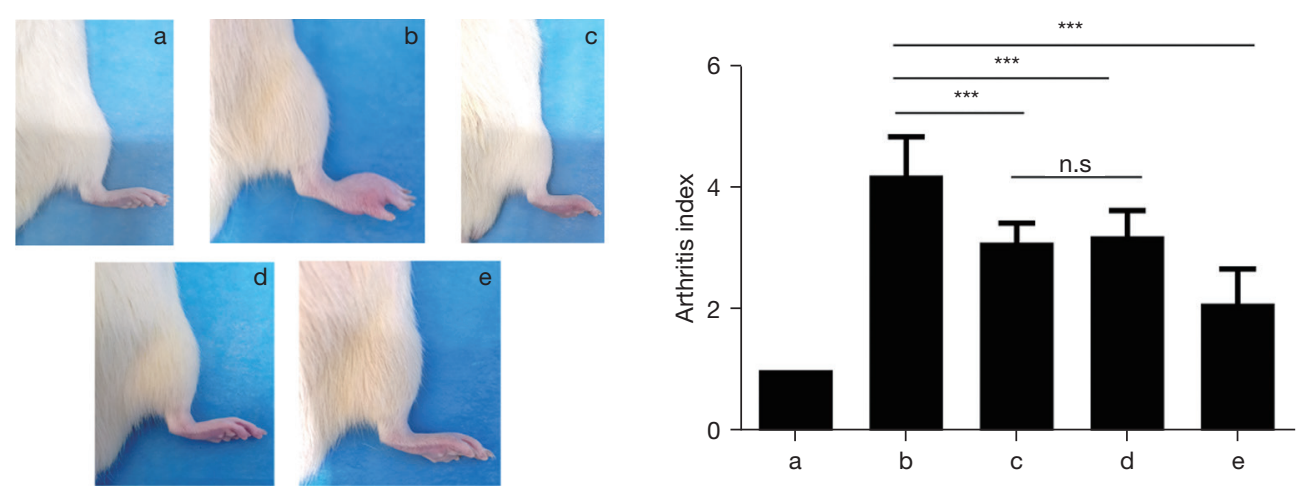

Figure 1 The method of Yiqi Yangyin Tongluo can attenuate the degree of inflammation in the ankle and paw in RA rats. a is the normal group, b is the model group, $\mathrm{c}$ is the methotrexate group, $\mathrm{d}$ is the Yiqi Yangyin Tongluo group, e is the combined group. ${ }^{* * *} \mathrm{P}<0.001(t$-test); n.s, $\mathrm{P}>0.05$ ( $t$-test).

Table 1 shows the fluorescent quantitative PCR primers used for ASIC1a, NLRP3, caspase 1, ASC, and $\beta$-actin.

\section{Statistical analysis}

SPSS 11.5 statistical software was used for data processing and statistical analysis, which was conducted using the t-test.

\section{Results}

\section{The effect of Yiqi Yangyin Tongluo on the inflammation of the feet of $R A$ rats}

To detect the effect of the method of Yiqi Yangyin Tongluo on the paw joints of RA rats, the RA rat model was established using FCA. RA rats were given daily intragastric administration of methotrexate (group c), compound of Yiqi Yangyin (group d), or compound of Yiqi Yangyin + methotrexate (group e). At the same time, for the normal control group (group a) and the RA model group (group b), sterilized water was administered daily. After 6 weeks of treatment, the rats in group a were in good mental state, normal eating and drinking, shiny coat color, active behavior and increased body weight; rats in group b were lethargic, increased food and water intake, decreased hair gloss, decreased behavioral activity, and decreased body weight compared with the normal group. Compared with group b, the rats in groups $\mathrm{c}, \mathrm{d}$, and e increased their body weight, improved their spirit, hair gloss, and behavioral activity, and drank less water than the model group. The results showed that compared with the model group, the swelling of the ankle joints of rats in the different administration groups recovered significantly (Figure 1A). By calculating the AI of rats, it was found that methotrexate (group c), compound of Yiqi Yangyin (group d), and compound of Yiqi Yangyin + methotrexate (group e) all had a significant rescue effect $(\mathrm{P}<0.001)$ on the ankle joints of RA rats, and there was no significant difference between methotrexate (group c) and compound of Yiqi Yangyin (group d, P >0.05, Figure 1B). Therefore, compound of Yiqi Yangyin does have a certain therapeutic effect on RA. 

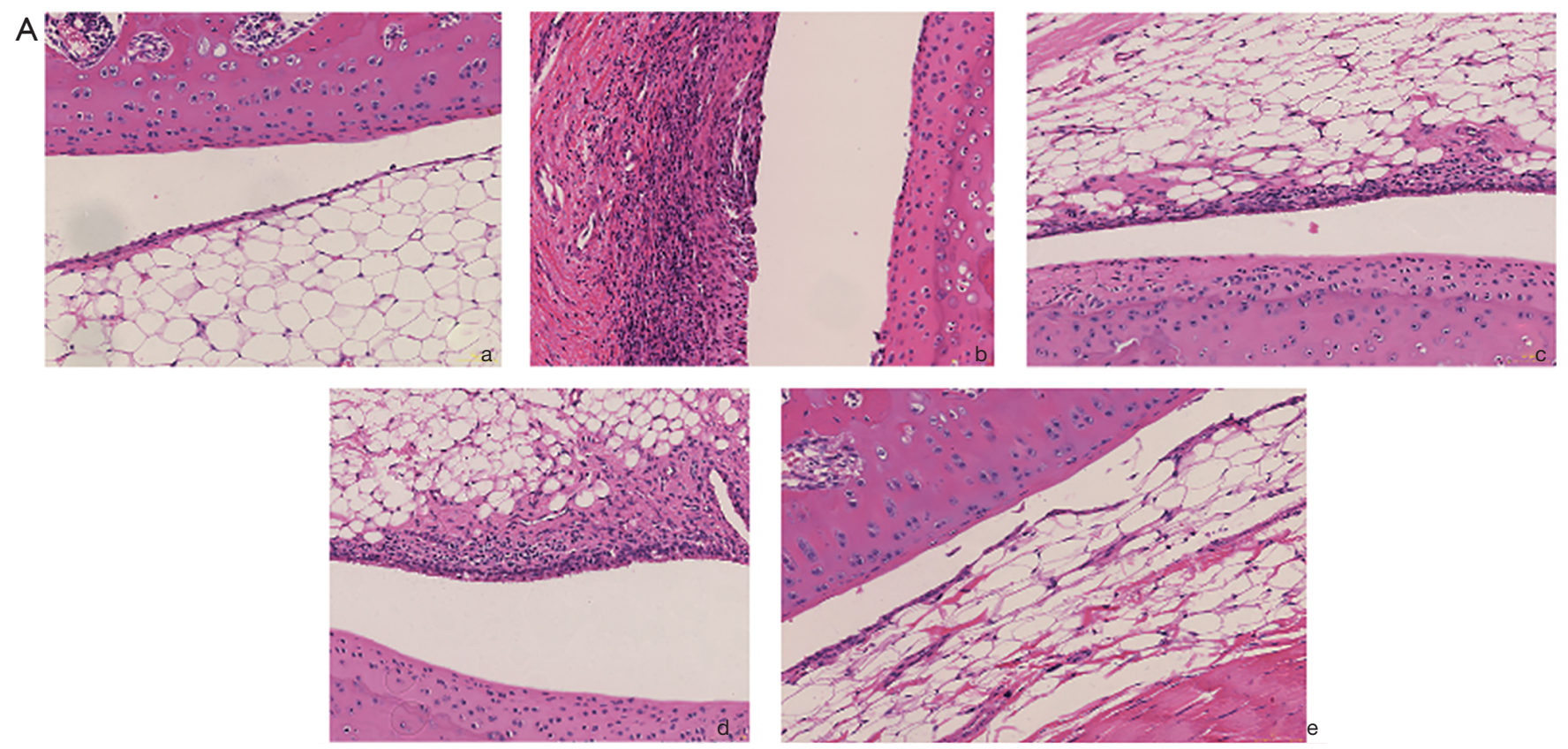

B
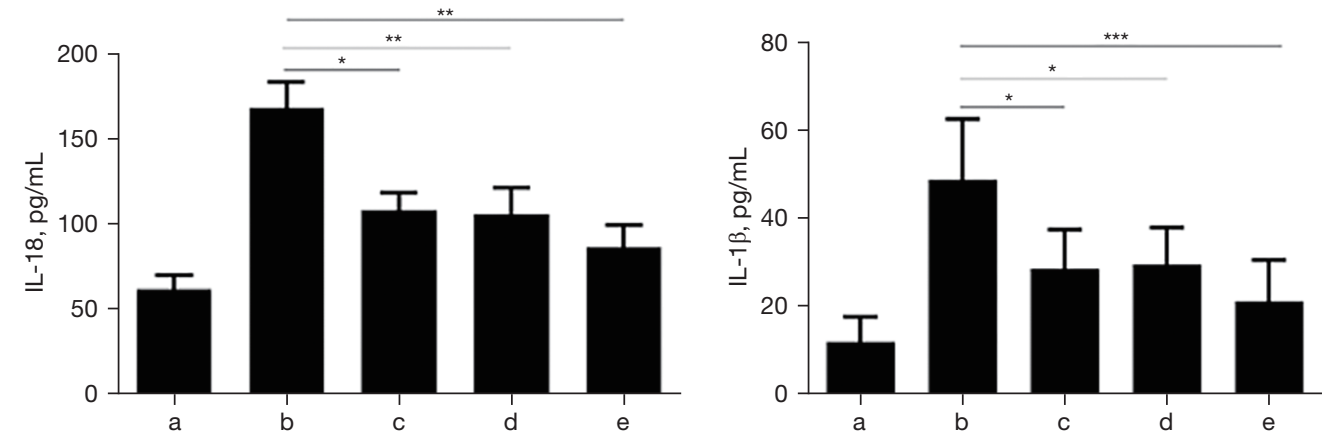

Figure 2 The method of Yiqi Yangyin Tongluo can lower the inflammation level in RA rats. (A) HE staining of the rat ankle joint $(\times 100)$; (B) serum IL-1 $\beta$ and IL-18 inflammatory factor levels. a is the normal group, $b$ is the model group, $\mathrm{c}$ is the methotrexate group, $\mathrm{d}$ is the Yiqi Yangyin Tongluo group, e is the combined group. ${ }^{* *} \mathrm{P}<0.001$ ( $t$-test), ${ }^{* *} \mathrm{P}<0.01$ ( $t$-test), ${ }^{*} \mathrm{P}<0.05$ ( $t$-test). IL-1 $\beta$, interleukin- $1 \beta ;$ IL-18, interleukin-18.

\section{The effect of Yiqi Yangyin Tongluo on inflammatory factors in $R A$ rats}

According to observations of pathological sections under light microscopy, there was no infiltration of inflammatory cells and hyperplasia of pannus in the joint subcutaneous soft tissue and synovial membrane of the normal control group (Figure $2 A$ ), but the rats in the model group showed obvious infiltration of inflammatory cells and proliferation of synovial cells (Figure 2A,2B). Compared with the model group, the infiltration of inflammatory cells and synovial hyperplasia in the soft tissues under the skin of the joints in RA rats treated with compound of Yiqi Yangyin was significantly reduced (Figure $2 A, 2 B$ ). At the same time, we also analyzed the inflammatory factors IL-18 and IL$1 \beta$ in chondrocytes and found that compared with the normal group, the inflammatory factors IL-18 and IL-1 $\beta$ in chondrocytes in the model group were significantly upregulated. However, the inflammatory factors in RA rats after administration treatment were down-regulated to varying degrees. Compared with the model group, the level of IL-18 in rats of the Yiqi Yangyin Tongluo group was significantly reduced $(\mathrm{P}<0.01)$, along with IL-1 $\beta(\mathrm{P}<0.05)$. Compared with the model group, the Yiqi Yangyin Tongluo group, and the methotrexate group, the combined group had the most significant reduction in the levels of IL-18 
and IL-1 $\beta$ (Figure $2 B$ ), which showed that after treatment with compound of Yiqi Yangyin, the inflammation level in RA rats was greatly relieved. Therefore, compound of Yiqi Yangyin has an excellent therapeutic effect on inflammation.

\section{The influence of the method of Yiqi Yangyin Tongluo on the ASIC1a/NLRP3 patbway}

To verify that compound of Yiqi Yangyin can regulate the inflammation level in RA rats through the ASIC1a/NLRP3 pathway, the knee joint tissues of the normal control group, model group, methotrexate group, Yiqi Yangyin Tongluo group, and combination group were used to detect the protein levels in RA rats. Immunofluorescence showed that the ACS and ASIC1a protein levels in RA rats in the methotrexate group, Yiqi Yangyin Tongluo group, and combination group were significantly lower than those in the model group (Figure 3A). NLRP3 and caspase 1 were also significantly down-regulated compared with the normal control group (Figure 3B). Furthermore, the mRNA levels of NLRP3, caspase 1, ACS, and ASIC1a were detected. The qPCR results showed that compared with the model group, the transcription levels of NLRP3, caspase 1, ACS, and ASIC1a in the methotrexate group, Yiqi Yangyin Tongluo group, and combination group RA rats were significantly down-regulated $(\mathrm{P}<0.01$, Figure $3 C)$. Compared with the methotrexate group and the Yiqi Yangyin Tongluo group, the combination group had the most significant down-regulation of NLRP3, caspase 1, ACS, and ASIC1a (Figure 3C). Also, we verified that the protein levels of NLRP3, caspase 1, ACS, and ASIC1a in the RA rats in the methotrexate group, the Yiqi Yangyin Tongluo group, and the combination group were also reduced compared with the normal control group. The above results demonstrated that the relieving effect of nourishing qi, nourishing yin, and dredging collaterals on inflammatory factors in RA rats was mediated by the ASIC1a/NLRP3 pathway.

\section{Discussion}

$\mathrm{RA}$ is a common and frequently occurring disease at present. Given its rapid progression, low compliance rate, high disability rate, and many other characteristics, it can seriously affect people's normal work and life. The incidence of RA with deficiency of both qi and yin is increasing year by year $(28,29)$. Previous studies have shown that the expression of inflammatory factors IL-18, IL- $1 \beta$, and TNF- $\alpha$ in articular cartilage cells of patients with RA due to deficiency of both qi and yin sharply increase (30). The ASIC1a/NLRP3 signaling pathway is a well-known inflammatory signaling transduction pathway $(31,32)$. Therefore, we speculated that the compound of Yiqi Yangyin, a traditional Chinese medicine for Yiqi Yangyin Tongluo, can regulate the level of inflammation in RA patients through the ASIC1a/ NLRP3 signaling pathway. Our results showed that the diseased tissues of the knee joint and the inflammation level in the body of RA rats treated with compound of Yiqi Yangyin showed greater improvement. At the same time, the transcription levels and protein levels of transcription factors NLRP3, ACS, ASIC1a, and pyroptosis factor caspase 1 in the ASIC1a/NLRP3 signaling pathway were significantly down-regulated. Therefore, compound of Yiqi Yangyin has a certain effect on RA with deficiency of both qi and yin.

As an anti-rheumatic drug, methotrexate is widely used in clinics (33). Studies have found that it has a certain regulatory effect on the apoptosis factors Bcl-2 and caspase 3 and the inflammatory factor TNF- $\alpha$ (34-36). In this study, we found through in vivo experiments that compound of Yiqi Yangyin can effectively reduce the knee joint inflammation of RA rats, and its combination with the western medicine methotrexate has significantly higher efficacy than methotrexate alone. This suggests that compound of Yiqi Yangyin and methotrexate have complementary effects. From the pathological tissue slices, we also clearly observed that the number of pannus in the cartilage tissue of the knee joint of RA rats in the combination group was less than that of the methotrexate and compound of Yiqi Yangyin groups alone. The results of qPCR also showed that the transcription levels of the transcription factors NLRP3, ACS, and ASIC1a of the ASIC1a/NLRP3 signaling pathway in the combination group were lower than those of the methotrexate and compound of Yiqi Yangyin groups alone. These results reveal the efficacy of compound of Yiqi Yangyin and compound of Yiqi Yangyin combined with anti-rheumatic western medicine in the treatment of RA patients. However, our study also has certain limitations. Although the study found that compound of Yiqi Yangyin has an inhibitory effect on the expression of ASIC1a/NLRP3 signaling pathway, the potential mechanism of the compound of Yiqi Yangyin improving chondrocyte pyroptosis in adjuvant arthritis rats still needs further research to verify.

In summary, our research confirms that the compound of Yiqi Yangyin Tongluo can effectively treat RA, and this treatment is mediated by the ASIC1a/NLRP3 signaling pathway. The results of this study show that compound 

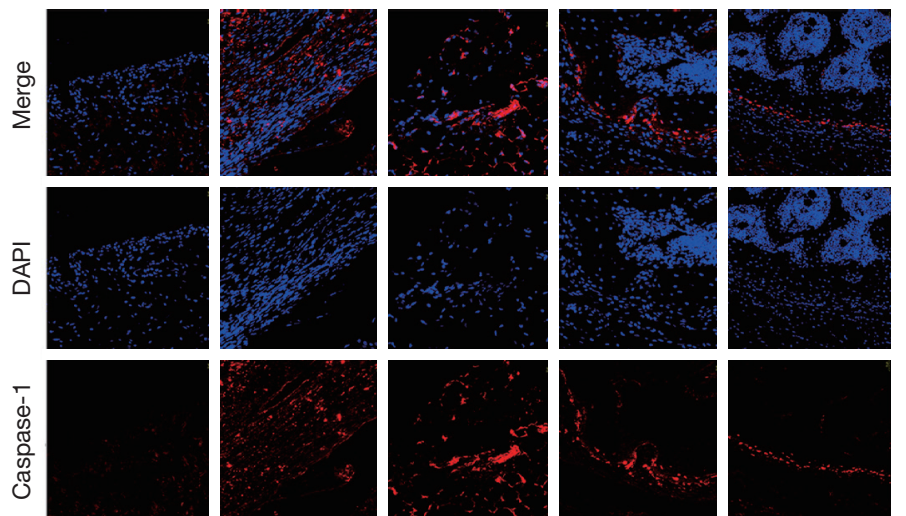

莡
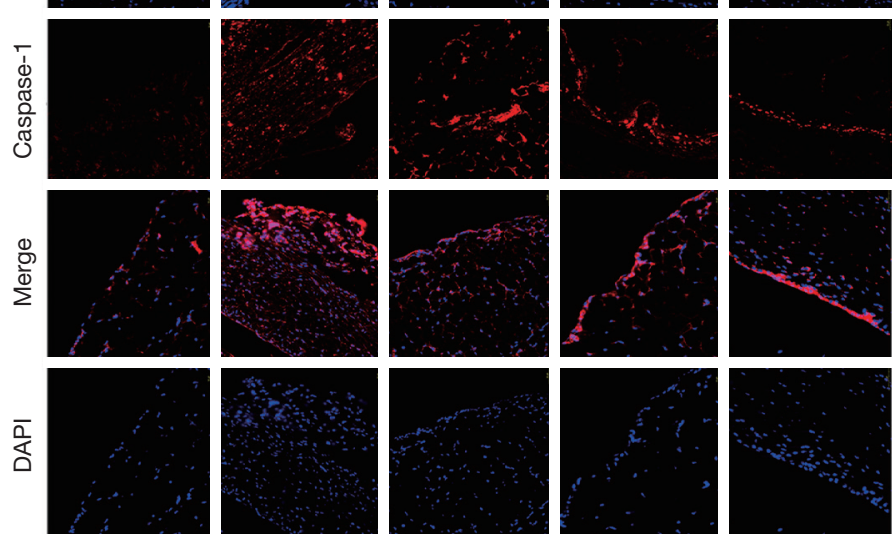
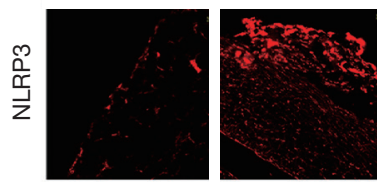

م
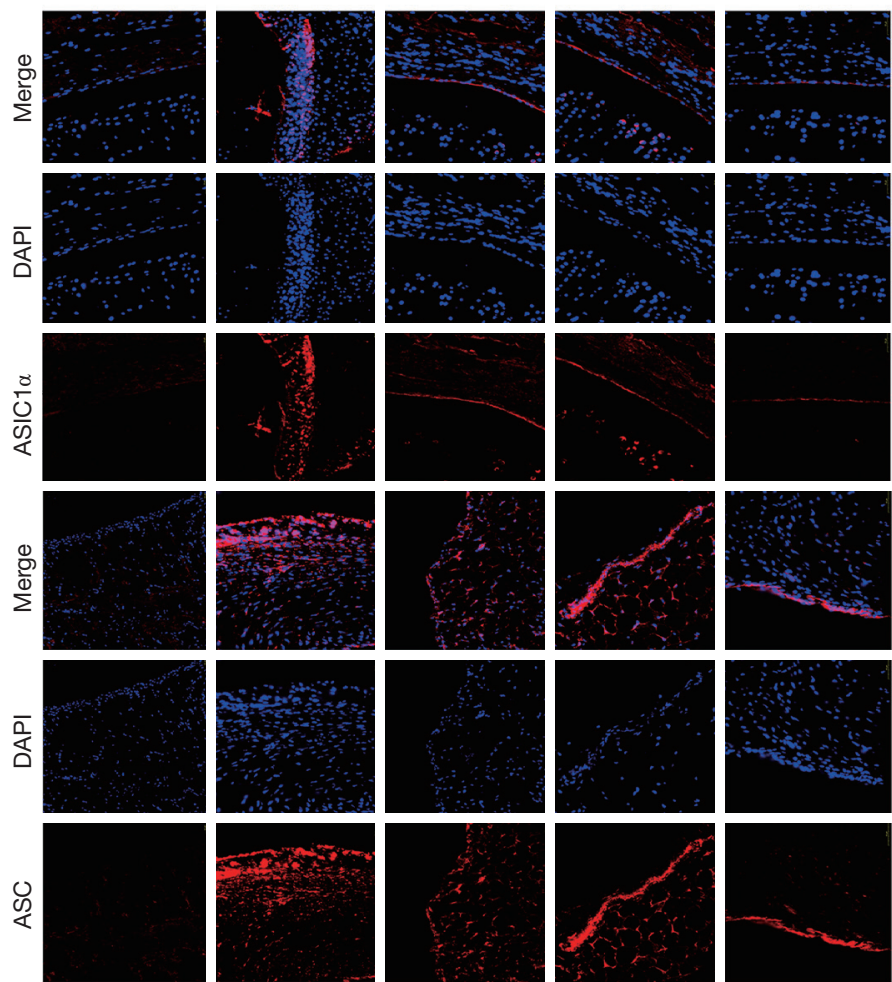

$\varangle$

๑
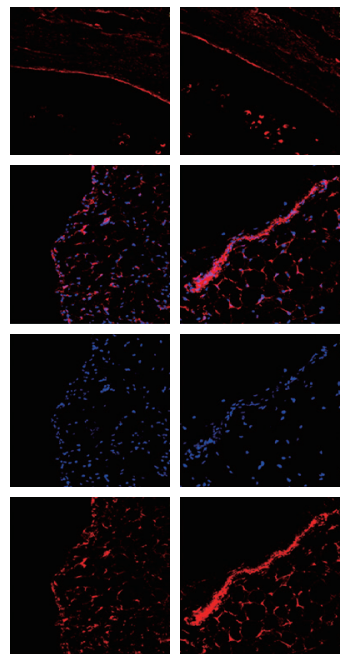

u

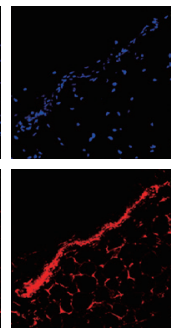

ð
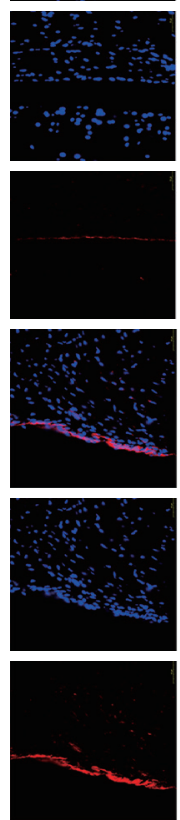

(

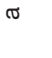


of Yiqi Yangyin, a traditional Chinese medicine for Yiqi Yangyin Tongluo, inhibits the activity of the ASIC1a/ NLRP3 signaling pathway, thereby reducing the secretion of inflammatory factors IL-18 and IL- $1 \beta$ and ultimately reducing the symptoms of RA rats. These results demonstrated that the ASIC1a/NLRP3 signaling pathway may be the underlying mechanism of the Chinese herbal method of Yiqi Yangyin Tongluo, and improves chondrocyte pyroptosis in rats with RA. These findings provide an effective basis for the clinical use of traditional Chinese medicine to treat RA.

\section{Acknowledgments}

Funding: This work was supported by Scientific Research Funding Project of the Education Department of Liaoning Province (L202041) and Natural Science Foundation of Liaoning Province (2021-BS-180).

\section{Footnote}

Reporting Checklist: The authors have completed the ARRIVE reporting checklist. Available at https://atm. amegroups.com/article/view/10.21037/atm-21-6822/rc

Data Sharing Statement: Available at https://atm.amegroups. com/article/view/10.21037/atm-21-6822/dss

Conflicts of Interest: All authors have completed the ICMJE uniform disclosure form (available at https://atm. amegroups.com/article/view/10.21037/atm-21-6822/coif). The authors have no conflicts of interest to declare.

Ethical Statement: The authors are accountable for all aspects of the work in ensuring that questions related to the accuracy or integrity of any part of the work are appropriately investigated and resolved. Experiments were performed under a project license (No. SCXK 2019-0008) granted by the Experimental Animals Ethics Committee of the Affiliated Hospital of Liaoning University of Traditional Chinese Medicine, in compliance with national and the Affiliated Hospital of Liaoning University of Traditional Chinese Medicine guidelines for the care and use of animals.

Open Access Statement: This is an Open Access article distributed in accordance with the Creative Commons Attribution-NonCommercial-NoDerivs 4.0 International License (CC BY-NC-ND 4.0), which permits the non- commercial replication and distribution of the article with the strict proviso that no changes or edits are made and the original work is properly cited (including links to both the formal publication through the relevant DOI and the license). See: https://creativecommons.org/licenses/by-nc-nd/4.0/.

\section{References}

1. Fu X, Li ZJ, Yang CJ, et al. The prevalence of depression in rheumatoid arthritis in China: A systematic review. Oncotarget 2017;8:53623-30.

2. Stump KL, Lu LD, Dobrzanski P, et al. A highly selective, orally active inhibitor of Janus kinase 2, CEP-33779, ablates disease in two mouse models of rheumatoid arthritis. Arthritis Res Ther 2011;13:R68.

3. Liu QS, Luo XY, Jiang H, et al. Salvia miltiorrhiza injection restores apoptosis of fibroblast-like synoviocytes cultured with serum from patients with rheumatoid arthritis. Mol Med Rep 2015;11:1476-82.

4. Pisetsky DS. Advances in the Treatment of Rheumatoid Arthritis: Costs and Challenges. N C Med J 2017;78:337-40.

5. Bi Y, Ye L, Mao Y, et al. Porous carbon supported nanoceria derived from one step in situ pyrolysis of Jerusalem artichoke stalk for functionalization of solutiongated graphene transistors for real-time detection of lactic acid from cancer cell metabolism. Biosens Bioelectron 2019;140:111271.

6. Khalaf W, Al-Rubaie HA, Shihab S. Studying anemia of chronic disease and iron deficiency in patients with rheumatoid arthritis by iron status and circulating hepcidin. Hematol Rep 2019;11:7708.

7. Zhao H, Zhang Y, Liu B, et al. Identification of Characteristic Autoantibodies Associated With Deficiency Pattern in Traditional Chinese Medicine of Rheumatoid Arthritis Using Protein Chips. Front Pharmacol 2019;10:755.

8. Cao W, Zhang HD, Liu HX, et al. Clinical observation of 50 cases of rheumatoid arthritis treated with Sishenjian. Journal of Beijing University of Traditional Chinese Medicine 2008;31: 490-493,498.

9. Zordan P, Rigamonti E, Freudenberg K, et al. Macrophages commit postnatal endothelium-derived progenitors to angiogenesis and restrict endothelial to mesenchymal transition during muscle regeneration. Cell Death Dis 2014;5:e1031.

10. Turesson C, Jacobsson LT, Matteson EL. Cardiovascular co-morbidity in rheumatic diseases. Vasc Health Risk Manag 2008;4:605-14. 
11. Cookson BT, Brennan MA. Pro-inflammatory programmed cell death. Trends Microbiol 2001;9:113-4.

12. Lim Y, Kumar S. A single cut to pyroptosis. Oncotarget 2015;6:36926-7.

13. Martinon F, Pétrilli V, Mayor A, et al. Gout-associated uric acid crystals activate the NALP3 inflammasome. Nature 2006;440:237-41.

14. Schorn C, Frey B, Lauber K, et al. Sodium overload and water influx activate the NALP3 inflammasome. J Biol Chem 2011;286:35-41.

15. Compan V, Baroja-Mazo A, López-Castejón G, et al. Cell volume regulation modulates NLRP3 inflammasome activation. Immunity 2012;37:487-500.

16. Sborgi L, Rühl S, Mulvihill E, et al. GSDMD membrane pore formation constitutes the mechanism of pyroptotic cell death. EMBO J 2016;35:1766-78.

17. Ystgaard MB, Sejersted Y, Løberg EM, et al. Early Upregulation of NLRP3 in the Brain of Neonatal Mice Exposed to Hypoxia-Ischemia: No Early Neuroprotective Effects of NLRP3 Deficiency. Neonatology 2015;108:211-9.

18. Vidal F, Gillibert A, Quillard M, et al. Does iron deficiency contribute to fatigue in patients with rheumatoid arthritis without anemia? Joint Bone Spine 2020;87:89.

19. Wang Q, Wang Q, Song XL, et al. Fear extinction requires ASIC1a-dependent regulation of hippocampal-prefrontal correlates. Sci Adv 2018;4:eaau3075.

20. Leng T, Lin J, Cottrell JE, et al. Subunit and frequencydependent inhibition of acid sensing ion channels by local anesthetic tetracaine. Mol Pain 2013;9:27.

21. Inoue $M$, Sato EF, Nishikawa $M$, et al. Mitochondrial generation of reactive oxygen species and its role in aerobic life. Curr Med Chem 2003;10:2495-505.

22. Lee GS, Subramanian N, Kim AI, et al. The calciumsensing receptor regulates the NLRP3 inflammasome through Ca2+ and cAMP. Nature 2012;492:123-7.

23. Murakami T, Ockinger J, Yu J, et al. Critical role for calcium mobilization in activation of the NLRP3 inflammasome. Proc Natl Acad Sci U S A 2012;109:11282-7.

24. Muñoz-Planillo R, Kuffa P, Martínez-Colón G, et al. $\mathrm{K}^{+}$ efflux is the common trigger of NLRP3 inflammasome activation by bacterial toxins and particulate matter. Immunity 2013;38:1142-53.

25. Hardy R, Cooper MS. Unravelling how glucocorticoids work in rheumatoid arthritis. Nat Rev Rheumatol 2018;14:566-7.

26. Park S, Shin J, Bae J, et al. SIRT1 Alleviates LPS-Induced IL-1 $\beta$ Production by Suppressing NLRP3 Inflammasome Activation and ROS Production in Trophoblasts. Cells 2020;9:728.
27. Sukhanov S, Higashi Y, Yoshida T, et al. The SGLT2 inhibitor Empagliflozin attenuates interleukin-17A-induced human aortic smooth muscle cell proliferation and migration by targeting TRAF3IP2/ROS/NLRP3/Caspase-1-dependent IL-1 $\beta$ and IL-18 secretion. Cell Signal 2021;77:109825.

28. Berardi S, Corrado A, Maruotti N, et al. Osteoblast role in the pathogenesis of rheumatoid arthritis. Mol Biol Rep 2021;48:2843-52.

29. Pradeepkiran JA. Insights of rheumatoid arthritis risk factors and associations. J Transl Autoimmun 2019;2:100012.

30. Cai LP, Zhou LJ, Lu SY, et al. Association of IL-18 promoter gene polymorphisms with rheumatoid arthritis: a meta-analysis. Mol Biol Rep 2014;41:8211-7.

31. Alehashemi S, Goldbach-Mansky R. Human Autoinflammatory Diseases Mediated by NLRP3-, Pyrin-, NLRP1-, and NLRC4-Inflammasome Dysregulation Updates on Diagnosis, Treatment, and the Respective Roles of IL-1 and IL-18. Front Immunol 2020;11:1840.

32. Li X, Liu S, Luo J, et al. Helicobacter pylori induces IL$1 \beta$ and IL-18 production in human monocytic cell line through activation of NLRP3 inflammasome via ROS signaling pathway. Pathog Dis 2015;73:ftu024.

33. Hayashi K, Sada KE, Asano Y, et al. Risk of higher dose methotrexate for renal impairment in patients with rheumatoid arthritis. Sci Rep 2020;10:18715.

34. Abdel-Wahab BA, Ali FEM, Alkahtani SA, et al. Hepatoprotective effect of rebamipide against methotrexate-induced hepatic intoxication: role of $\mathrm{Nrf} 2 /$ GSK-3 $\beta$, NF- $\kappa \beta$-p65/JAK1/STAT3, and PUMA/Bax/Bcl2 signaling pathways. Immunopharmacol Immunotoxicol 2020;42:493-503.

35. Ryan C, Menter A. Psoriasis and cardiovascular disorders. G Ital Dermatol Venereol 2012;147:179-87.

36. Yiang GT, Chen TY, Chen C, et al. Antioxidant vitamins promote anticancer effects on low-concentration methotrexate-treated glioblastoma cells via enhancing the caspase-3 death pathway. Food Sci Nutr 2021;9:3308-16.

(English Language Editor: C. Betlazar-Maseh)

Cite this article as: Zhao Y, Chen Y, Wang Z, Peng C, Li Q, Wu J, Wang L, Liu D, Yue Y, Qing Q, Sun P, Liang L, Yu H, Ju X, Li L, Wang E, Gao M, Yu J, Ma T. The method of Yiqi Yangyin Tongluo can attenuate the pyroptosis of rheumatoid arthritis chondrocytes through the ASIC1a/NLRP3 signaling pathway. Ann Transl Med 2022;10(3):145. doi: 10.21037/atm21-6822 